\title{
CRONOLOGÍA DE ERUPCIÓN DE LOS DIENTES PERMANENTES EN NIÑOS Y NIÑAS DE 5, 6 Y 7 AÑOS *
}

\author{
${ }^{1}$ Laura Marcela Ayala Chiquillo, ${ }^{1}$ Adriana Marcela Arias Rueda, ${ }^{1}$ Astrid Carolina Gutiérrez Díaz, ${ }^{2}$ Martha Juliana Rodríguez Gómez. \\ ${ }^{1}$ Estudiante X semestre F. de Odontología U. Santo Tomás. ${ }^{2}$ Odontóloga U. Javeriana, Especialista en Odontopediatría y Ortodoncia Preventiva \\ U. CES, Docente U. Santo Tomás.
}

\author{
Autor responsable de correspondencia: Martha Juliana Rodríguez G. \\ Correo electrónico: marthajuro@yahoo.com
}

\section{RESUMEN}

Objetivos: Determinar la edad de erupción del primer molar (6), el incisivo central (1) y el incisivo lateral (2) permanente y establecer la secuencia de erupción en niños(as) de 5, 6 y 7 años de edad.

Materiales y métodos: Se realizó un estudio observacional descriptivo de corte transversal con una muestra total de 900 niños(as) colombianos. Fueron examinados con espejo bucal y sonda bajo luz adecuada. Adicionalmente, se registró su peso y talla para establecer el Índice de Masa Corporal. Se calcularon la media, mediana, rango, varianza y desviación estándar para todos los datos. Se aplicó coeficiente de correlación de Sperman o de Pearson y T student ó U de Mann Whitney.

Resultados: Se observó que los dientes más frecuentes fueron el incisivo central inferior derecho en 544 niños(as) y el incisivo central inferior izquierdo (543). La secuencia de erupción varío según el sexo y la erupción de los dientes permanentes fue más temprana en los niños que en las niñas. El primer diente en erupcionar en los niños fue el incisivo central inferior derecho (41) y en las niñas, el primer molar inferior derecho (46).

Conclusión: El primer diente en erupcionar fue el incisivo central inferior a los 6 años 11 meses. El último fue el incisivo lateral superior (7 años 5 meses). La secuencia de erupción tanto para niños como para las niñas, en el maxilar superior fue 6, 1, 2. En el maxilar inferior fue 1, 6, 2 en las niños y 6, 1, 2 en las niñas. [Ayala LM, Arias AM, Gutiérrez AC, Rodríguez MJ. Cronología de erupción de los dientes permanentes en niños y niñas de 5, 6 y 7 años. Ustasalud 2010; 9: 26 - 33]

Palabras clave: Erupción, Dentición permanente, Cronología, Secuencia.

\section{CHRONOLOGY OF THE ERUPTION OF PERMANENT TEETH IN CHILDREN OF 5, 6 AND 7 YEARS OF AGE}

\section{ABSTRACT}

Objective: To determine de age of eruption of the first permanent molar (6), central (1) and lateral (2) incisors and to stablish the eruption sequence in a group of children ranging from 5,6 and 7 years of age.

Methods: An observational descriptive cross sectional study based on a sample of 900 Colombian children was done. They were examined with mouth mirror and probe under adequate illumination for the status of the eruption of the teeth mentioned. Additionally, their weight and height was registered to calculate the Body Mass Index. Means, modes, medians and standard deviation were determined for all data. Bivariate analyses were performed by Student's $t$ and Mann Whitney U test.

Results: The lower right central incisor (41) and the lower left central incisor (31) were the teeth that appeared more frequently in 544 and 543 children, respectively. The sequence of eruption varied according to sex and the eruption of permanent teeth was earlier in boys than girls by a minimum difference. The first tooth to erupt in males was the lower right central incisor (41) and the first lower right molar (46) in females.

Conclusion: The first tooth erupted at 6 years 11 months (lower central incisor) and the latest was the upper lateral incisor at 7 years 5 months. The eruption sequence both in males and females was $6,1,2$ for the maxilla. For the mandible, it was $1,6,2$ for males and $6,1,2$ for the females.

Key words: Eruption, Permanent dentition, Chronology, Sequence.

Recibido para publicación: 25 de abril de 2010. Aceptado para publicación: 5 de junio de 2010.

${ }^{*}$ Grupo de Investigación CYDUSTA 


\section{INTRODUCCIÓN}

Conocer la cronología y la secuencia de erupción de la dentición permanente es esencial para planear un tratamiento odontológico. La información que se pueda obtener sobre la erupción dental es indispensable para realizar un diagnóstico preciso, un plan de tratamiento adecuado y, en odontología forense, para establecer la edad cronológica de niños con registro de nacimiento no conocido. ${ }^{1-6}$ En la población colombiana, hay pocos reportes publicados sobre la cronología de la erupción de los dientes permanentes. ${ }^{7,8}$ Por tal motivo, se manejan resultados de estudios poblacionales de otros lugares geográficos para comparar los hallazgos encontrados los niños(as) colombianos (Tabla 1). ${ }^{9-23}$

Los estudios sobre la cronología de erupción dental aportan importantes datos sobre los procesos biológicos que son la base en el desarrollo de la dentición, ${ }^{24,25}$ sin embargo, estos estudios presentan diferentes metodologías (clínicos, radiográficos), en algunos no se establece claramente los criterios de inclusión y exclusión, los rangos de edad son amplios, las poblaciones evaluadas son heterogéneas, la precisión de los métodos de valoración dental son diferentes así como su análisis estadístico.9-23,26,27

Algunos estudios han encontrado que los primeros dientes en erupcionar son los primeros molares inferiores, mientras que otros han observado que el primer diente en erupcionar es el incisivo central inferior. ${ }^{9-23}$ Se han generado distintas opiniones referentes a las causas que originan un adelanto o un retraso en la erupción normal de los dientes. ${ }^{28,29}$ Estas alteraciones requieren distintos tratamientos según la edad del individuo y su diagnóstico precoz mejora el pronóstico del tratamiento a realizar.

Conocedor de esta problemática, el grupo de investigación Crecimiento y Desarrollo (CYDUSTA), ha desarrollado algunos trabajos sobre las anomalías dentales que podrían afectar la erupción y sobre la cronología de erupción. ${ }^{26,30}$ Las recomendaciones de estos trabajos han dado pie a realizar una investigación con una muestra más homogénea con un rango de edades más pequeño. Por lo tanto, el objetivo de esta investigación fue determinar la edad de erupción del primer molar (6), el incisivo central (1) y el incisivo lateral (2) permanente y establecer la secuencia de erupción en niños(as) de 5, 6 y 7 años de edad de la ciudad de Bucaramanga y su área metropolitana.

\section{MATERIALES Y MÉTODOS}

Se realizó un estudio observacional descriptivo de corte transversal, cuyo universo estuvo integrado por niños y niñas de 5,6 y 7 años de edad de la ciudad de Bucaramanga y su área metropolitana. El calculo del tamaño de muestra se hizo con nivel de confianza del $99 \%$, poder $80 \%$, relación expuesto, no expuesto 1 a 1 , prevalencia de alteraciones en la erupción de laterales de los niños del $7 \%$ y de las niñas del $26 \%$ por lo cual se consideraron 278 escolares en edades de 5, 6 y 7 años, para un total de 834 escolares. Al considerar un potencial de atricion del $10 \%$, se evaluaron trescientos niños y niñas de 5 años, trescientos niños y niñas de 6 años, y trescientos niños y niñas de 7 años. En total, 900 sujetos (300 por cada grupo de edad).

El tipo de muestra fue por conveniencia, los criterios de inclusión fueron:

- Niños y niñas de 5, 6 y 7 años de edad sistémicamente sanos.

- Niños y niñas que vivieran en la ciudad de Bucaramanga y su área metropolitana.

Entre los criterios de exclusión estaban:

- Niños y niñas con antecedentes de lesiones traumáticas y exodoncias prematuras.

- Presencia de anomalías dentales (número y forma). Se consideraron variables dependientes: número de dientes presentes para la edad de 5, 6 y 7 años; variables independientes: edad, sexo, estrato socioeconómico, índice de masa corporal.

Previo a la prueba piloto, se realizó la calibración interexaminador con un experto y la calibración intraexaminador. Los valores Kappa obtenidos se consideraron de muy buena reproducibilidad $(0,84$ a 1,0$)$.

Para la selección de la muestra se tuvieron en cuenta los colegios que tenían convenios interinstitucionales con la Universidad Santo Tomás. Se dio prioridad a los colegios que tenían la mayor cantidad de niños y niñas dentro del rango de edad necesario para el estudio. Se entregó el consentimiento informado para los padres donde se les explicaba las razones del estudio y los beneficios que podían obtener si los niños participaban en la investigación. El consentimiento informado de los padres debía estar firmado para que el niño(a) ingresara al grupo de estudio.

Para la revisión oral, el niño sentó en una silla y su cabeza se apoyó sobre las piernas del examinador; se realizó un examen clínico en el que se usaron todas las medidas de bioseguridad, espejo bucal y sonda periodontal Hu-Friedy ${ }^{\mathbb{R}}$ para observar si estaban presentes o no los dientes a evaluar (primer molar, incisivo central e incisivo lateral permanente). Además, se registró la talla y el peso para calcular el Índice de Masa Corporal (IMC). 
ARTÍCULO ORIGINAL

Tabla 1. Edad de erupción del primer molar, incisivo central y lateral permanente.

\begin{tabular}{|c|c|c|c|c|c|c|c|c|}
\hline \multirow[b]{2}{*}{ POBLACIÓN } & \multirow[b]{2}{*}{ AUTORES } & \multirow[b]{2}{*}{ GÉNERO } & \multicolumn{3}{|c|}{ MAXILAR SUPERIOR } & \multicolumn{3}{|c|}{ MAXILAR INFERIOR } \\
\hline & & & $\begin{array}{l}\text { Incisivo } \\
\text { central }\end{array}$ & $\begin{array}{c}\text { Incisivo } \\
\text { lateral }\end{array}$ & $\begin{array}{l}\text { Primer } \\
\text { molar }\end{array}$ & $\begin{array}{r}\text { Incisivo } \\
\text { central }\end{array}$ & $\begin{array}{l}\text { Incisivo } \\
\text { lateral }\end{array}$ & $\begin{array}{l}\text { Primer } \\
\text { molar }\end{array}$ \\
\hline \multirow{2}{*}{ Spanish } & \multirow{2}{*}{$\begin{array}{l}\text { Hernández M, } \\
\text { Espasa E, Boj JR. }\end{array}$} & $\mathrm{xx}$ & 6,89 & 7,37 & 5,96 & 6,00 & 7,26 & 6,09 \\
\hline & & $X Y$ & 7,17 & 8,21 & 6,28 & 6,29 & 7,52 & 6,13 \\
\hline \multirow{2}{*}{ Flemish } & \multirow{2}{*}{$\begin{array}{l}\text { Leroy R, Bogaerts } \\
\text { K, Lesaffre E, } \\
\text { Declerck D. }{ }^{14}\end{array}$} & $\mathrm{XX}$ & 6,85 & 7,84 & 6,14 & 6,11 & 7,07 & 6,17 \\
\hline & & $X Y$ & 7,08 & 8,25 & 6,31 & 6,27 & 7,36 & 6,33 \\
\hline \multirow{2}{*}{$\begin{array}{l}\text { Northerm } \\
\text { Ireland }\end{array}$} & \multirow{2}{*}{$\begin{array}{c}\text { Kochhar R, } \\
\text { Richardson A. }{ }^{15}\end{array}$} & $x x$ & 7,09 & 8,05 & 6,40 & 6,27 & 7,40 & 6,29 \\
\hline & & $\mathrm{XY}$ & 7,17 & 8,26 & 6,40 & 6,31 & 7,44 & 6,37 \\
\hline \multirow{2}{*}{ Mongoloid } & \multirow{2}{*}{ Orner G. ${ }^{19}$} & $\mathrm{xx}$ & 6,70 & 7,81 & 6,04 & 6,20 & 7,26 & 6,04 \\
\hline & & $X Y$ & 7,04 & 8,01 & 7,04 & 6,37 & 7,04 & 6,87 \\
\hline \multirow{2}{*}{ Manchester } & \multirow{2}{*}{$\begin{array}{l}\text { Miller J, Hobson P, } \\
\text { Gaskell TJ. }{ }^{13}\end{array}$} & $\mathrm{xx}$ & 7,08 & 8,00 & 6,15 & 6,22 & 7,45 & 5,92 \\
\hline & & $\mathrm{XY}$ & 7,30 & 8,40 & 6,23 & 6,15 & 7,68 & 6,13 \\
\hline \multirow{2}{*}{ Australian } & \multirow{2}{*}{$\begin{array}{l}\text { Diamanti J, } \\
\text { Townsend GC. }{ }^{16}\end{array}$} & $\mathrm{xx}$ & 7,17 & 8,24 & 6,57 & 6,38 & 7,47 & 6,42 \\
\hline & & $\mathrm{XY}$ & 7,43 & 8,61 & 6,71 & 6,63 & 7,77 & 6,63 \\
\hline \multirow{3}{*}{$\begin{array}{l}\text { Kelantan, } \\
\text { North- } \\
\text { Eastern, } \\
\text { Malasya }\end{array}$} & \multirow{3}{*}{$\begin{array}{l}\text { Nizam A, Naing L, } \\
\text { Mokhtar N. } .^{20}\end{array}$} & & & & & & & \\
\hline & & $\begin{array}{l}X X \\
X Y\end{array}$ & $\begin{array}{l}7,09 \\
7,17\end{array}$ & $\begin{array}{l}8,05 \\
8,26\end{array}$ & $\begin{array}{l}6,40 \\
6,40\end{array}$ & $\begin{array}{l}6,27 \\
6,31\end{array}$ & $\begin{array}{l}7,40 \\
7,44\end{array}$ & $\begin{array}{l}6,29 \\
6,37\end{array}$ \\
\hline & & & & & & & & \\
\hline \multirow{2}{*}{$\begin{array}{l}\text { Athens } \\
\text { (Greece) }\end{array}$} & \multirow{2}{*}{$\begin{array}{l}\text { Wedl JS, Danias } \\
\text { R, Schmelzle R, } \\
\text { Fiedrich RE.21 }\end{array}$} & $x X$ & 6,72 & 7,65 & 6,06 & 6,18 & 6,88 & 6,00 \\
\hline & & $\mathrm{XY}$ & 6,77 & 7,98 & 6,01 & 6,09 & 7,05 & 6,08 \\
\hline
\end{tabular}

XX: niñas XY: niños

Posterior al examen, se realizó una breve charla educativa con el niño y se entregó un informe escrito sobre el estado de su dentición. Al despedir el paciente se entregó un incentivo por su participación. Así mismo, se realizaron charlas sobre higiene oral y enseñanza de cepillado.

\section{Análisis estadístico}

Los registros obtenidos se digitaron en Excel por duplicado, se verificó la calidad de la digitación mediante la rutina Validate para exportar al paquete estadístico SPSS $18 .{ }^{31,32}$

En el análisis univariado se calcularon medidas de resumen según la naturaleza de las variables; para las cualitativas proporciones y para las cuantitativas medidas de resumen de tendencia central (media y mediana) y de dispersión (rango, varianza y desviación estándar). Para el análisis bivariado se consideró como variable de salida número de dientes presentes que se cruzó con variables cuantitativas para lo cual se aplicó coeficiente de correlación, de Sperman o de Pearson y para las cualitativas prueba t Student ó $\mathrm{U}$ de Mann Whitney.

\section{Consideraciones éticas}

Todos los procedimientos siguieron las recomendaciones de la resolución 008430 de 1993 del Ministerio de Salud de la República de Colombia, en su título II artículo 11 del capítulo I; esta investigación es de riesgo mínimo ya que se llevarán a cabo procedimientos de rutina como un examen odontológico, donde se tendrá en cuenta el respeto a la dignidad, protección de los derechos humanos, confidencialidad de los datos obtenidos, libertad de participación en el estudio, manteniendo así la integridad del individuo.

\section{RESULTADOS}

Se evaluó un total de 900 niños en edades entre 5, 6 y 7 años. De éstos, 359 (39,9\%) fueron mujeres. La distribución por edades fue de 181 (33,5\%) niños para el grupo de 5 años, $186(34,4 \%)$ niños para el de 6 y 174 $(32,2 \%)$ para el de 7 . Se evaluaron tanto colegios públicos como privados siendo mayor la población de colegios públicos (98,6\%). Así mismo, los niños(as) pertenecían a todos los estratos, el más frecuente fue el estrado 2 con 408 (45,3\%) niños(as) (Tabla 2). 
Tabla 2. Distribución por frecuencia y porcentaje de las variables sociodemográficas.

\begin{tabular}{lcc}
\hline Variable & & $\mathbf{n}(\%)$ \\
\hline Sexo & Masculino & $541(60,1)$ \\
& Femenino & $359(39,9)$ \\
Colegio & & \\
& Público & $887(98,6)$ \\
& Privado & $13(1,4)$ \\
Estrato & & \\
& 1 & $45(9,4)$ \\
& 2 & $373(41,4)$ \\
& 3 & $29(3,2)$ \\
& 4 & $3(0,3)$ \\
& 5 & $2(0,2)$ \\
\hline
\end{tabular}

En relación con los dientes presentes en los tres grupos de edad, se observó que los dientes más frecuentes fueron el incisivo central inferior derecho en $544(60,4 \%)$ niños(as) y el incisivo central inferior izquierdo en $543(60,3 \%)$. Con estos datos se deduce que la secuencia de erupción para la totalidad de la muestra fue: $41,31,46,36,26,16,11,21,42,32,22$ y 12 (Tabla 3).

En el sexo masculino, el primer diente permanente en erupcionar fue el incisivo central inferior derecho a los 6 años 11 meses y el último fue el incisivo lateral superior izquierdo a los 7 años 5 meses. De acuerdo con las edades evaluadas, la secuencia de erupción en los niños fue: 41,31 , $46,36,26,16,32,42,11,21,12$ y 22 . Por otro lado, en las niñas el primer diente en erupcionar fue el primer molar inferior derecho a la edad de 6 años 11 meses, y el último el incisivo lateral superior derecho a los 7 años y cinco meses (Tabla 4 y 5$)$.

Tabla 3. Frecuencia de dientes presentes en la muestra evaluada.

\begin{tabular}{cccccc}
\hline Número del diente & $\begin{array}{c}\text { Presente } \\
\mathbf{n}(\%)\end{array}$ & $\begin{array}{c}\text { Ausente } \\
\mathbf{n}(\%)\end{array}$ & Número del diente & $\begin{array}{c}\text { Presente } \\
\mathbf{n}(\%)\end{array}$ & $\begin{array}{c}\text { Ausente } \\
\mathbf{n}(\%)\end{array}$ \\
\hline 16 & $488(54,2)$ & $412(45,8)$ & 26 & $490(54,4)$ & $410(45,6)$ \\
12 & $81(9)$ & $819(91)$ & 22 & $84(9,3)$ & $816(90,7)$ \\
11 & $281(31,2)$ & $619(68,8)$ & 21 & $268(29,8)$ & $632(70,2)$ \\
41 & $544(60,4)$ & $356(39,6)$ & 31 & $543(60,3)$ & $357(39,7)$ \\
42 & $250(27,8)$ & $650(72,2)$ & 32 & $243(27)$, & $657(73)$ \\
46 & $538(59,8)$ & $362(40,2)$ & 36 & $535(59,4)$ & $365(40,6)$ \\
\hline
\end{tabular}

Tabla 4. Edad promedio de erupción en el sexo masculino (años y meses).

\begin{tabular}{|c|c|c|c|c|c|c|c|}
\hline \multicolumn{5}{|c|}{ CUADRANTE DERECHO } & \multicolumn{3}{|c|}{ CUADRANTE IZQUIERDO } \\
\hline $\begin{array}{l}\text { Número del } \\
\text { diente }\end{array}$ & $\begin{array}{c}\text { Edad } \\
\text { promedio }\end{array}$ & $\begin{array}{l}\text { Límite } \\
\text { inferior }\end{array}$ & $\begin{array}{l}\text { Límite } \\
\text { superior }\end{array}$ & $\begin{array}{l}\text { Número del } \\
\text { diente }\end{array}$ & $\begin{array}{c}\text { Edad } \\
\text { promedio }\end{array}$ & $\begin{array}{l}\text { Límite } \\
\text { inferior }\end{array}$ & $\begin{array}{l}\text { Límite } \\
\text { superior }\end{array}$ \\
\hline 41 & $\begin{array}{c}6,91 \\
(6 a, 11 \mathrm{~m})\end{array}$ & $\begin{array}{c}6,85 \\
(6 a, 10 \mathrm{~m})\end{array}$ & $\begin{array}{c}6,98 \\
(6 a, 12 \mathrm{~m})\end{array}$ & 31 & $\begin{array}{c}6,91 \\
(6 a, 11 \mathrm{~m})\end{array}$ & $\begin{array}{c}6,85 \\
(6 a, 10 \mathrm{~m})\end{array}$ & $\begin{array}{c}6,98 \\
(6 a, 12 \mathrm{~m})\end{array}$ \\
\hline 46 & $\begin{array}{c}6,94 \\
(6 a, 11 \mathrm{~m})\end{array}$ & $\begin{array}{c}6,88 \\
(6 \mathrm{a}, 11 \mathrm{~m})\end{array}$ & $\begin{array}{c}7,01 \\
(7 \mathrm{a}, 0 \mathrm{~m})\end{array}$ & 36 & $\begin{array}{c}6,95 \\
(6 \mathrm{a}, 11 \mathrm{~m})\end{array}$ & $\begin{array}{c}6,89 \\
(6 a, 11)\end{array}$ & $\begin{array}{c}7,01 \\
(7 \mathrm{a}, 0 \mathrm{~m})\end{array}$ \\
\hline 16 & $\begin{array}{c}6,99 \\
(6 a, 12 \mathrm{~m})\end{array}$ & $\begin{array}{c}6,92 \\
(6 a, 11 \mathrm{~m})\end{array}$ & $\begin{array}{c}7,05 \\
(7 \mathrm{a}, 1 \mathrm{~m})\end{array}$ & 26 & $\begin{array}{c}6,98 \\
(6 a, 12 \mathrm{~m})\end{array}$ & $\begin{array}{c}6,92 \\
(6 a, 11 \mathrm{~m})\end{array}$ & $\begin{array}{c}7,05 \\
(7 \mathrm{a}, 1 \mathrm{~m})\end{array}$ \\
\hline 42 & $\begin{array}{c}7,15 \\
(7 a, 2 m)\end{array}$ & $\begin{array}{c}7,07 \\
(7 \mathrm{a}, 1 \mathrm{~m})\end{array}$ & $\begin{array}{c}7,23 \\
(7 a, 3 \mathrm{~m})\end{array}$ & 32 & $\begin{array}{c}7,14 \\
(7 \mathrm{a}, 2 \mathrm{~m})\end{array}$ & $\begin{array}{c}7,06 \\
(7 \mathrm{a}, 1 \mathrm{~m})\end{array}$ & $\begin{array}{c}7,23 \\
(7 \mathrm{a}, 3 \mathrm{~m})\end{array}$ \\
\hline 11 & $\begin{array}{c}7,16 \\
(7 a, 2 m)\end{array}$ & $\begin{array}{c}7,08 \\
(7 \mathrm{a}, 1 \mathrm{~m})\end{array}$ & $\begin{array}{c}7,23 \\
(7 a, 3 \mathrm{~m})\end{array}$ & 21 & $\begin{array}{c}7,18 \\
(7 a, 2 \mathrm{~m})\end{array}$ & $\begin{array}{c}7,11 \\
(7 \mathrm{a}, 1 \mathrm{~m})\end{array}$ & $\begin{array}{c}7,26 \\
(7 a, 3 \mathrm{~m})\end{array}$ \\
\hline 12 & $\begin{array}{c}7,43 \\
(7 a, 5 \mathrm{~m})\end{array}$ & $\begin{array}{c}7,32 \\
(7 a, 4 \mathrm{~m})\end{array}$ & $\begin{array}{c}7,54 \\
(7 a, 6 \mathrm{~m})\end{array}$ & 22 & $\begin{array}{c}7,44 \\
(7 a, 5 \mathrm{~m})\end{array}$ & $\begin{array}{c}7,32 \\
(7 \mathrm{a}, 4 \mathrm{~m})\end{array}$ & $\begin{array}{c}7,55 \\
(7 \mathrm{a}, 7 \mathrm{~m})\end{array}$ \\
\hline
\end{tabular}


Tabla 5. Edad promedio de erupción en el sexo femenino (años y meses).

\begin{tabular}{|c|c|c|c|c|c|c|c|}
\hline \multicolumn{5}{|c|}{ CUADRANTE DERECHO } & \multicolumn{3}{|c|}{ CUADRANTE IZQUIERDO } \\
\hline $\begin{array}{l}\text { Número } \\
\text { del diente }\end{array}$ & $\begin{array}{c}\text { Edad } \\
\text { promedio }\end{array}$ & $\begin{array}{l}\text { Límite } \\
\text { inferior }\end{array}$ & $\begin{array}{l}\text { Límite } \\
\text { superior }\end{array}$ & $\begin{array}{l}\text { Número del } \\
\text { diente }\end{array}$ & $\begin{array}{c}\text { Edad } \\
\text { promedio }\end{array}$ & $\begin{array}{l}\text { Límite } \\
\text { inferior }\end{array}$ & $\begin{array}{l}\text { Límite } \\
\text { superior }\end{array}$ \\
\hline 46 & $\begin{array}{c}6,92 \\
(6 a, 11 \mathrm{~m})\end{array}$ & $\begin{array}{c}6,83 \\
(6 a, 10 \mathrm{~m})\end{array}$ & $\begin{array}{c}7,02 \\
(7 a, 0 \mathrm{~m})\end{array}$ & 36 & $\begin{array}{c}6,95 \\
(6 \mathrm{a}, 11 \mathrm{~m})\end{array}$ & $\begin{array}{c}6,86 \\
(6 a, 10 \mathrm{~m})\end{array}$ & $\begin{array}{c}7,04 \\
(7 \mathrm{a}, 1 \mathrm{~m})\end{array}$ \\
\hline 41 & $\begin{array}{c}6,94 \\
(6 a, 11 \mathrm{~m})\end{array}$ & $\begin{array}{c}6,85 \\
(6 a, 10 \mathrm{~m})\end{array}$ & $\begin{array}{c}7,03 \\
(7 a, 0 \mathrm{~m})\end{array}$ & 31 & $\begin{array}{c}6,96 \\
(6 a, 11 \mathrm{~m})\end{array}$ & $\begin{array}{c}6,87 \\
(6 a, 8 \mathrm{~m})\end{array}$ & $\begin{array}{c}7,05 \\
(7 \mathrm{a}, 1 \mathrm{~m})\end{array}$ \\
\hline 16 & $\begin{array}{c}7,03 \\
(7 a, 0 \mathrm{~m})\end{array}$ & $\begin{array}{c}6,94 \\
(6 a, 11 \mathrm{~m})\end{array}$ & $\begin{array}{c}7,12 \\
(7 \mathrm{a}, 1 \mathrm{~m})\end{array}$ & 26 & $\begin{array}{c}7,04 \\
(7 a, 0 m)\end{array}$ & $\begin{array}{c}6,94 \\
(6 a, 11 \mathrm{~m})\end{array}$ & $\begin{array}{c}7,13 \\
(7 \mathrm{a}, 2 \mathrm{~m})\end{array}$ \\
\hline 11 & $\begin{array}{c}7,21 \\
(7 a, 3 \mathrm{~m})\end{array}$ & $\begin{array}{c}7,11 \\
(7 \mathrm{a}, 1 \mathrm{~m})\end{array}$ & $\begin{array}{c}7,31 \\
(7 a, 4 m)\end{array}$ & 21 & $\begin{array}{c}7,24 \\
(7 a, 3 \mathrm{~m})\end{array}$ & $\begin{array}{c}7,14 \\
(7 \mathrm{a}, 2 \mathrm{~m})\end{array}$ & $\begin{array}{c}7,34 \\
(7 \mathrm{a}, 4 \mathrm{~m})\end{array}$ \\
\hline 42 & $\begin{array}{c}7,24 \\
(7 a, 3 \mathrm{~m})\end{array}$ & $\begin{array}{c}7,14 \\
(7 a, 2 \mathrm{~m})\end{array}$ & $\begin{array}{c}7,34 \\
(7 a, 4)\end{array}$ & 32 & $\begin{array}{c}7,21 \\
(7 a, 3 \mathrm{~m})\end{array}$ & $\begin{array}{c}7,11 \\
(7 \mathrm{a}, 1 \mathrm{~m})\end{array}$ & $\begin{array}{c}7,32 \\
(7 \mathrm{a}, 4 \mathrm{~m})\end{array}$ \\
\hline 12 & $\begin{array}{c}7,45 \\
(7 a, 5 \mathrm{~m})\end{array}$ & $\begin{array}{c}7,32 \\
(7 a, 4 \mathrm{~m})\end{array}$ & $\begin{array}{c}7,57 \\
(7 \mathrm{a}, 7 \mathrm{~m})\end{array}$ & 22 & $\begin{array}{c}7,41 \\
(7 a, 5 \mathrm{~m})\end{array}$ & $\begin{array}{c}7,26 \\
(7 a, 3 \mathrm{~m})\end{array}$ & $\begin{array}{c}7,56 \\
(7 \mathrm{a}, 7 \mathrm{~m})\end{array}$ \\
\hline
\end{tabular}

Al relacionar el IMC promedio y la erupción dental para el sexo masculino se observó que el incisivo central inferior derecho ya se encontraba emergiendo en los niños que presentaban un IMC de 16,51. En los niños con un IMC de 18,69, se observó que el último diente en erupcionar fue el incisivo lateral superior derecho (Tabla 6). Por otro lado, las niñas que presentaron un IMC promedio de 16,49 ya presentaban el incisivo central inferior derecho y cuan- do su IMC subia a 17,31 se observaba el incisivo lateral superior izquierdo (Tabla 7).

La secuencia de erupción para el grupo de niños con relación al IMC promedio se presento de la siguiente manera: $41,16,31,36,46,26,11,21,42,32,22$, 12; para el grupo de niñas se presentó el siguiente orden: $41,31,36,46,26,16,42,11,21,32,12,22$ (Tabla 6 y 7 ).

Tabla 6. Relación entre el IMC promedio y la erupción dental para el sexo masculino.

\begin{tabular}{|c|c|c|c|c|c|c|c|}
\hline \multicolumn{5}{|c|}{ CUADRANTE DERECHO } & \multicolumn{3}{|c|}{ CUADRANTE IZQUIERDO } \\
\hline $\begin{array}{l}\text { Número del } \\
\text { diente }\end{array}$ & $\begin{array}{c}\mathrm{IMC} \\
\left(\mathrm{Kg} / \mathrm{m}^{2}\right)\end{array}$ & $\begin{array}{l}\text { Límite } \\
\text { inferior }\end{array}$ & $\begin{array}{c}\text { Límite } \\
\text { superior }\end{array}$ & $\begin{array}{l}\text { Número del } \\
\text { diente }\end{array}$ & $\begin{array}{c}\mathrm{IMC} \\
\left(\mathrm{Kg} / \mathrm{m}^{2}\right)\end{array}$ & $\begin{array}{l}\text { Límite } \\
\text { inferior }\end{array}$ & $\begin{array}{c}\text { Límite } \\
\text { superior }\end{array}$ \\
\hline 41 & 16,51 & 16,36 & 16,66 & 31 & 16,93 & 16,66 & 17,20 \\
\hline 16 & 16,58 & 16,43 & 16,74 & 36 & 16,98 & 16,71 & 17,26 \\
\hline 46 & 17,01 & 16,73 & 17,28 & 26 & 17,04 & 16,76 & 17,32 \\
\hline 11 & 17,47 & 17,04 & 17,90 & 21 & 17,49 & 17,04 & 17,93 \\
\hline 42 & 17,73 & 17,25 & 18,20 & 32 & 17,77 & 17,28 & 18,26 \\
\hline 12 & 18,69 & 17,47 & 19,91 & 22 & 18,43 & 17,39 & 19,47 \\
\hline
\end{tabular}


Tabla 7. Relación entre el IMC promedio y la erupción dental para el sexo femenino.

\begin{tabular}{cccccccc}
\hline & \multicolumn{3}{c}{ CUADRANTE DERECHO } & \multicolumn{3}{c}{ CUADRANTE IZQUIERDO } \\
$\begin{array}{c}\text { Número del } \\
\text { diente }\end{array}$ & $\begin{array}{c}\text { IMC } \\
\left(\mathbf{K g} / \mathbf{m}^{2}\right)\end{array}$ & $\begin{array}{c}\text { Límite } \\
\text { inferior }\end{array}$ & $\begin{array}{c}\text { Límite } \\
\text { superior }\end{array}$ & $\begin{array}{c}\text { Número del } \\
\text { diente }\end{array}$ & $\begin{array}{c}\text { IMC } \\
\mathbf{( K g / \mathbf { m } ^ { 2 } )}\end{array}$ & $\begin{array}{c}\text { Límite } \\
\text { inferior }\end{array}$ & $\begin{array}{c}\text { Límite } \\
\text { superior }\end{array}$ \\
\hline $\mathbf{4 1}$ & 16,49 & 16,16 & 16,81 & $\mathbf{3 1}$ & 16,53 & 16,20 & 16,86 \\
$\mathbf{4 6}$ & 16,60 & 16,28 & 16,92 & $\mathbf{3 6}$ & 16,60 & 16,28 & 16,92 \\
$\mathbf{1 6}$ & 16,66 & 16,30 & 17,02 & $\mathbf{2 6}$ & 16,63 & 16,28 & 16,99 \\
$\mathbf{4 2}$ & 17,10 & 16,57 & 17,63 & $\mathbf{2 1}$ & 17,17 & 16,67 & 17,68 \\
$\mathbf{1 1}$ & 17,14 & 16,64 & 17,64 & $\mathbf{3 2}$ & 17,21 & 16,66 & 17,75 \\
$\mathbf{1 2}$ & 17,30 & 16,67 & 17,92 & $\mathbf{2 2}$ & 17,31 & 16,69 & 17,93 \\
\hline
\end{tabular}

\section{DISCUSIÓN}

Después de evaluar a un grupo de 900 niños(as) de las ciudad de Bucaramanga y su área metropolitana se observó que la secuencia de erupción varía según el sexo; así mismo, se encontró que los dientes inferiores erupcionan primero que los superiores como lo reportan la mayoría de estudios revisados. ${ }^{9-23}$

La secuencia de erupción de los primeros molares e incisivos permanentes de acuerdo con los resultados de este estudio, tanto para niños como para niñas, fue: $41,31,46,36,26,16,11,21,42,32,22$ y 12. Estos datos se consideran confiables debido a que no se tuvo en cuenta los niños(as) con pérdida prematura de dientes deciduos. Diversos estudios han reportado que uno de los factores que más podría afectar la erupción de dientes permanentes es la ausencia o perdida prematura del diente primario. $^{4,13,28}$

Schour y Masller afirmaron que la erupción de los incisivos y el primer molar comprendía la primera fase de erupción, aseguraban que el primer molar erupciona antes que el incisivo central en un 83.3\% de los sujetos y el incisivo central inferior antes que el primer molar permanente, en sólo un $5.8 \%$. En un $10.4 \%$ de los sujetos estos dos dientes erupcionaron en el mismo rango de edad. ${ }^{33}$ Estos hallazgos son diferentes a los observados en este estudio ya que los primeros dientes en erupcionar fueron los incisivos centrales inferiores, seguidos de los primeros molares.

Los resultados concuerdan con varios estudios en los que también se encontró que, en los niños, el primer diente permanente en erupcionar fue el incisivo central inferior. ${ }^{14,15,19,20}$ Sin embargo, en el sexo femenino, el primer molar inferior fue el primer diente permanente en erupcionar lo que también se ha observado. ${ }^{13,19,21}$

Se ha reportado que para este grupo de edad, los últimos dientes en erupcionar son los incisivos laterales superiores. ${ }^{10-16}$ Estos hallazgos concuerdan con los resultados obtenidos en el presente estudio.

Diversos investigadores han sugerido que la erupción de los dientes permanentes sucede primero en las niñas que en los niños..$^{10,13,14,19,20}$ Sin embargo, en el presente estudio, la erupción dental se inició primero en los niños. Posiblemente, este hallazgo fue debido a que la población de niños y niñas no fue homogénea, se evaluaron más sujetos del sexo masculino que del femenino.

Es importante anotar que los niños examinados no tenían antecedentes de exodoncias o pérdidas prematuras de sus piezas dentales. Se ha demostrado que la pérdida prematura del diente deciduo afecta la erupción del diente permanente. ${ }^{1,13,29}$

Por otro lado, Diamanti y Townsend afirmaron que el tiempo de emergencia era similar tanto en la hemiarcada derecha como en la izquierda. ${ }^{16}$ Leroy y colaboradores encontraron que los dientes contralaterales erupcionaban con una diferencia máxima de 15 meses, lo que consideraron como un tiempo relativamente corto. ${ }^{14}$ En el presente estudio se observó que los dientes de la hemiarcada contraria erupcionaban casi al mismo tiempo siendo la diferencia máxima de 12 meses. 
Gupta y colaboradores sugirieron que las diferencias la constitución corporal de las personas pueden ocasionar variaciones en los tiempos de erupción de los dientes permanentes. ${ }^{22}$ Hernández y colaboradores registraron talla y peso pero no lo relacionaron con la secuencia de erupción. ${ }^{10}$ Por otro lado, Sánchez - Pérez y colaboradores encontraron que los niños con sobrepeso, medido a través del Índice de Masa Corporal (IMC), tenían más dientes permanentes erupcionados. ${ }^{34}$ Así mismo lo sugiere Hilgers y colaboradores..$^{35}$ En este estudio, también se encontró el mismo hallazgo, a mayor valor del IMC mayor cantidad de dientes en niños y niñas.

En la Universidad Santo Tomás Bucaramanga, ya se había realizado un estudio sobre la cronología de erupción. ${ }^{26}$ Sin embargo, los resultados no son comparables ya que se evaluaron 100 radiografias panorámicas en un rango de edad de 5 a 14 años, su muestra fue muy amplia para el grupo de edad y no fue homogénea.

Finalmente, se puede decir que la secuencia de erupción observada en este estudio difiere de la propuesta por Lo y Moyers ya que se encontró que los incisivos centrales inferiores erupcionan antes que los primeros molares inferiores. Otros estudios confirman esta secuencia. ${ }^{14-16,20}$

Una de las debilidades del estudio fue no contar con el mismo número de niñas que de niños. Sin embargo, al realizar el diseño metodológico del estudio no se pensó que la diferencia numérica entre sexos pudiera afectar porque de acuerdo con la literatura revisada, la mayoría de estudios tenían tamaños de muestra diferentes de niños y niñas, y no lo reportaban como debilidad. ${ }^{10-22}$

El tamaño de muestra total es importante y que al ser un estudio de observación clínica con criterios bien establecidos como la no inclusión de sujetos que presentaran exodoncias prematuras, anomalías dentales y compromiso sistémico sugiere que los datos encontrados sean creibles y relevantes.

Es de anotar que dentro de la muestra no hubo niños(as) pertenecientes a alguna etnia. Por lo tanto, los datos observados no se pueden comparar con estudios que reflejaran etnias o grupos indígenas. ${ }^{36}$

Esta investigación es la primera de una serie de tres estudios que intentan conocer la cronología y la secuencia de erupción de los dientes permanentes. Los objetivos que se trazaron se cumplieron y la hipótesis planteada en la que se enunciaba que el primer molar precedía la erupción del incisivo central inferior no coincidió.

\section{Conclusiones}

- El primer diente en erupcionar fue el incisivo central inferior a los 6 años 11 meses. El último fue el incisivo lateral superior a los 7 años 5 meses.

- Se observó que la secuencia de erupción de acuerdo con la edad es:

- Niños: maxilar superior: $6,1,2$; maxilar inferior: $1,6,2$.

- Niñas: maxilar superior: $6,1,2$; maxilar inferior: $6,1,2$.

- Esta secuencia es la misma en los niños al tener en cuenta el IMC. Sin embargo, en las niñas la secuencia cambia en el maxilar inferior al relacionarlo con el IMC siendo la secuencia en el maxilar inferior: 1, 6, 2 .

\section{Agradecimientos}

Al rector, directivos, profesores y niños(as) del Instituto Técnico Superior Dámaso Zapata quienes colaboraron con este estudio.

\section{BIBLIOGRAFÍA}

1. Sanabria AD, Jiménez DM, Gutiérrez TI, Pilonieta G. Factores que influyen en la cronología de erupción de los dientes permanentes. Ustasalud 2006; 5: 132 - 136.

2. Burdi AR, Moyers RE. Desarrollo de la dentición y la oclusión. En: Moyers RE. Manual de Ortodoncia. Argentina: Editorial Médica Panamericana S.A.; 1988. p. 117 - 122.

3. Cárdenas D, Arias MI, Baquero X. Desarrollo de la Oclusión. En: Odontología Pediátrica. 4ta. Ed. Medellín: Corporación para la Investigación Biológica; 2009. p. $352-358$.

4. Van der Linden F. Ortodontia. Desenvolvimento da dentição. Quintessence Editora Ltda; 1986.

5. Maber M, Liversigde HM, Hector MP. Accuracy of age estimation of radiographic methods developing teeth. Forensic Sci Int 2006; 159: S68 - S73.

6. Espina de Ferreira A, Ferreira J, Céspedes M, Barrios F, Ortega A, Maldonado Y. Empleo de la edad dental y la edad ósea para el cálculo de la edad cronológica con fines forenses, en niños escolares con alteraciones en el estado nutricional, en Maracaibo, Estado Zulia. Estudio preliminar. Acta Odontol Venez 2007; 45: 433 - 439.

7. Estrada MI, Espinosa MM, Carvajal LA. Cronología de erupción de la dentición permanente. CES Odontología 1987; 1: $14-18$.

8. González MC, Martínez M, Caballero L, Salamanca M, Gamboa LF, Lafaurie G. Desarrollo de la dentición permanente en una población de niños colombianos con y sin labio y paladar fisurado. Odontol Pediatr 2009; 8: 4 - 8.

9. Lo RT, Moyers RE. Studies in the etiology and prevention of malocclusion. I. The sequence of eruption of the permanent dentition. Am J Orthod 1953: 39: 460 - 467.

10. Hernández M, Espasa E, Boj JR. Eruption chronology of the permanent dentition in Spanish children. J Clin Pediatr Dent 2008; 32: $347-350$.

11. Lee SE, Lee SH, Lee JY, Park HK, Kim YK. Age estimation of Korean children based on dental maturity. Forensic Sci Int 2008; 178: $125-131$. 
12. Moslemi M. An epidemiological survey of the time and sequence of eruption of permanent teeth in $4-15$ year-olds in Tehran, Iran. Int J Paediatr Dent 2004; 14: 432 - 438.

13. Miller J, Hobson P, Gaskell TJ. A serial study of the chronology of exfoliation of deciduous teeth and eruption of permanent teeth. Arch Oral Biol 1965; 10: $805-818$.

14. Leroy R, Bogaerts K, Lesaffre E, Declerck D. The emergence of permanent teeth in Flemish children. Community Dent Oral Epidemiol 2003; 31: 30 - 39.

15. Kochhar R, Richardson A. The chronology and sequence of eruption of human permanent teeth in Northen Ireland. Int J Paediatr Dent 1998; 8: 243 - 252.

16. Diamanti J, Townsed GC. New standards for permanent tooth emergence in Australian children. Aust Dent J 2003; 48: $39-42$.

17. Plasensia E, García-Izquierdo F, Puente-Rodríguez M. Edad de emergencia y secuencias polimórficas de la dentición permanente en una muestra de población de Asturias. RCOE [en línea] 2005 Feb [fecha de acceso: enero de 2009]; 10: 31 - 42. URL disponible en: http://scielo.isciii. es/scielo.php?script $=$ sci_arttext\&pid $=$ S1138-123X2005 000100003\&lng $=$ es\&nrm $=$ iso.

18. Mugonzibwa EA, Kuijpers-Jagtman AM, Laine-Alava MT, Van' T Hof MA. Emergence of permanent teeth in Tanzania children. Community Dent Oral Epidemiol 2002; 30: $455-462$

19. Orner G. Eruption of permanent teeth in Mongoloid children and their sibs. J Dent Res 1973; 52: $1202-1208$

20. Nizam A, Naing L, Mokhtar N. Age and sequence of eruption of permanent teeth in Kelantan, North-Eastern, Malasya. Clin Oral Investig 2008; 7: 222 - 225.

21. Wedl JS, Danias S, Schmelzle R, Friedrich RE. Eruption times of permanent teeth in children and young adolescents in Athens (Greece). Clin Oral Investig 2005; 9: 131 -134 .

22. Gupta R. Sivapathasundharam B, Einstein A. Eruption age of permanent mandibular first molars and central incisors in the South Indian population. Indian J Dent Res 2007; 18: $186-189$.

23. Knott VB, Meredith HV. Statistics on eruption of the permanent dentition from serial data for North American white children. Angle Orthod 1966; 36: 68 - 79 .

24. Parner ET, Heidmann JM, Kjaer I, Vaeth M, Poulsen S. Biological interpretation of the correlation of emergence times of permanent teeth. J Dent Res 2002; 81: 451 - 454.

25. Steedle RJ, Profit WR. The pattern and control of eruptive to movement. Am J Orthod 1988; 87: 56 - 66.

26. Sanabria $A D$, Jiménez DM, Gutiérrez TI, Rodríguez MJ. Identificación de la cronología de erupción de dientes permanentes en una población escolarizada de Bucaramanga [Trabajo de Grado]. Bucaramanga: Universidad Santo Tomás; 2007.

27. Lew KK. The prediction of eruption sequence from panoramic radiographs. J Dent Child 1992; 56: 346- 349.

28. Suri L, Gagari E, Vastardis H. Delayed tooth eruption: Pathogenesis, diagnosis, and treatment. A literature review. Am J Orthod Dentofacial Orthop 2004; 126: 432 - 445.

29. McDonald RE, Avery DR. Erupción de los dientes: factores locales, sistémicos y congénitos que influyen en el proceso. En: Odontología Pediátrica y del Adolescente. 6ta. Ed. Madrid: Mosby/Doyma Libros; 1995. p. 179-185.

30. Angarita L, Rodríguez MJ. Estudio retrospectivo de las anomalías dentarias en niños de 4 a 14 años. Revista de la Academia Colombiana de Odontología Pediátrica. 2007; 5: $9-14$

31. Microsoft Office Excel. Microsoft Corporation, 2007.

32. SPSS Statistics 18, 2009.

33. Schour I, Massler M. Development of the human dentition. J Am Dent Assoc 1941; 28: 1153 - 1160.

34. Sánchez-Pérez L, Irigoyen ME, Zepeda M. Dental caries, tooth eruption timing and obesity: a longitudinal stuy in a group of Mexican schoolchildren. Acta Odontol Scand 2010; 68: $57-64$.

35. Hilgers KK, Akridge M, Scheetz JP, Kinane DE. Childhood obesity and dental development. Pediatr Dent 2006; 28: $18-22$.

36. Morón A, Santana Y, Pirona M, Rivera L, Rincón MC, Pirela A. Cronología y secuencia de erupción de dientes permanentes en escolares Wayúu. Acta Odontol Venez 2006; 44.

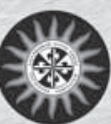
UniverSidAd Santo Tomas PRIMER CLAUSTRO UNIVERSITARIO DE COLOMBIA B U C A R A M N G A

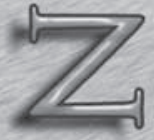

La facultad de odontología, orientada por el pensamiento de Santo Tomás, pretende formar Odontólogos integrales $y / 0$ especialistas a través de parámetros humanísticos, éticos, científicos, biotecnológicos, investigativos

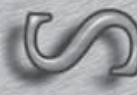
y sociales, como recurso humano capaz de intervenir con éxito en el

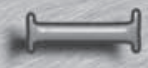
proceso dinámico de la salud y la enfermedad, en el individuo, la familia y la comunidad. 